\title{
Diversidad de Polychaeta (Annelida) en el intermareal de los esteros del Santuario Nacional los Manglares de Tumbes, Perú
}

\section{Diversity of Polychaeta (Annelida) in the intertidal estuaries from Santuario Nacional los Manglares de Tumbes, Peru}

\section{Rossana Cabanillas*, Orlando Advíncula y Carlos Gutiérrez}

\author{
Laboratorio de Ecología Acuática, Facultad de Ciencias Biológicas, Universidad Nacional Mayor de San Marcos, Apartado 1898, Lima 100, Perú \\ *Autora para correspondencia \\ Email Rossana Cabanillas: rossanacinthia@gmail.com \\ Email Orlando Advíncula: orlando_ad@outlook.com \\ Email Carlos Gutiérrez: cargutierrezr@gmail.com
}

\begin{abstract}
Resumen
Se estudia la diversidad de anélidos poliquetos asociados al biotopo intermareal del manglar del Santuario Nacional los Manglares de Tumbes, Perú. El muestreo se realizó en dos sitios, uno cercano a la desembocadura y el otro distante aproximadamente $6 \mathrm{~km}$ rio arriba. En cada lugar se establecieron dos estaciones fijas, una asociada a zonas bajas del intermareal con presencia de Anadara tuberculosa (ZBI) y otra asociada a la zona altas del intermareal con presencia de Ucides occidentalis (ZBI). Los muestreos se repitieron en diciembre del 2012 y marzo del 2013. El número total de especies de poliquetos registrado fue 57, la composición de especies no fue similar entre las asociaciones y entre las estaciones fijas. Las estaciones asociadas a ZBI tuvieron un mayor número de especies, abundancias e índices de diversidad mayores que las estaciones asociadas a ZAI. En cuanto a los grupos tróficos, las estaciones asociadas a ZAI tuvieron 4 grupos mientras que las estaciones asociadas a ZBI tuvieron 6 grupos tróficos. En la poliquetofauna asociada a ZBI dominaron, en número de especie los carnívoros mientras que en la asociada a ZAI, fueron los carnívoros y los alimentadores de depósito superficial, asimismo dominaron en abundancia los alimentadores de depósito sub superficial. El análisis multivariado demostró diferencia significativa en la estructura y composición trófica de las asociaciones, con mayor dispersión y variabilidad en el ZAI.
\end{abstract}

Palabras clave: Poliquetos; Ecosistema de manglar; Biotopos, Ucides occidentalis; Anadara tuberculosa.

\section{Abstract}

The diversity of annelids polychaete associated with intertidal mangrove biotope of the Santuario Nacional los Manglares de Tumbes is studied. Sampling was conducted at two sites, one near the river mouth and the other distant about $6 \mathrm{~km}$ upstream. In each place, two fixed stations were established, one associated to high intertidal zone with the presence of Anadara tuberculosa (ZBI) and other associated to low intertidal zone with the presence of Ucides occidentalis (ZAI). The samples were taken in December 2012 and March 2013. The total number of species registered was 57 species composition was not similar between associations and between fixed stations. The stations associated to ZBI had more number of species abundances and indices greater diversity than to ZAI. As trophic groups, the stations associated to ZAI had four groups whereas stations associated to ZBI had 6 trophic groups. The poliquetofauna associated with ZBI dominated in number of species, the carnivores while poliquetofauna associated to ZAI, was carnivores and surface deposit feeders also dominated in abundance subsurface deposit feeders. Multivariate analysis showed significant differences in the structure and trophic composition of partnerships, with greater dispersion and variability in the ZAI.

Keywords: Polychaeta; Ecosystem of Mangrove; biotope; Ucides occidentalis; Anadara tuberculosa.

Citación:

Cabanillas R., O. Advíncula y C. Gutiérrez. 2016. Diversidad de Polychaeta (Annelida) en el intermareal de los esteros del Santuario Nacional los Manglares de Tumbes, Perú. Revista peruana de biología 23(2): 117 - 126 (Agosto 2016). doi: http://dx.doi.org/10.15381/rpb.v23i2.12383
Información sobre los autores:

R. C. diseño de metodología, toma de muestra, identificación de poliquetos, análisis de datos, redacción, discusión, revisión. O. A. identificación de poliquetos, análisis de datos, revisión. C. G. identificación de poliquetos, análisis estadístico, grupos tróficos, discusión y revisión. Los autores no incurren en conflictos de intereses.

\section{Fuentes de financiamiento:}

Proyecto "Impacto de la Variabilidad y Cambio Climático en el Ecosistema de Manglares de Tumbes" -IDRC \#106714-001 (CCW). 


\section{Introducción}

El Santuario Nacional los Manglares de Tumbes (SNLMT) fue creado el 7 de marzo de 1988 con el DS Nº18-88-AG, para contribuir a la protección del Bosque de manglar así como la gran biodiversidad asociada a él (INRENA, 2007; Lacerda, 1993).

El ecosistema de manglar es caracterizado por un bosque de árboles tolerantes a la salinidad, y que se desarrollan en relación a un estuario (Duke 1993, Gilman et al. 2008, Macnae 1968). En el borde de los canales del estuario del rio Zarumilla localizado en el SNLMT se puede observar el biotopo de Rhizophora mangle y $R$. harrisoni así como también el efecto intermareal con flujo y reflujo ocasionado por las mareas altas y bajas. Es en esta zona donde se desarrollan organismos bentónicos de importancia económica (Echevarría \& Sarabia, 1993, INRENA 2007).

En la zona intermareal podemos encontrar, una zona baja con la presencia de Anadara tuberculosa (concha negra) y una zona alta con la presencia de Ucides occidentalis (cangrejo del manglar), ambos constituyen dos de los invertebrados de importancia económica en el ambiente de los manglares (INRENA 2007, Peña 1970). Ademá, en estas zonas encontramos una comunidad de organismos bentónicos cuya estructura y composición aún se mantiene en estudio (Takahashi \& Martínez 2015).

La macrofauna bentónica del ecosistema de manglar es un componente poco estudiado en Perú (v. g. Vargas 1993), en particular los poliquetos los cuales resaltan por su rol ecológico (Hutchings \& Recher 1982) y como indicadores del impacto antropogénico (Giangrande et al. 2005, Metcalfe \& Glasby 2008).

El presente trabajo describe la diversidad de poliquetos del macrobentos intermareal, presente en el biotopo de manglar del Santuario Nacional Los Manglares de Tumbes, analizando la estructura de especies y gremios tróficos de los ensambles según su asociación con Anadara tuberculosa y Ucides occidentalis y además si se ven afectadas por la cercanía o lejanía al mar.

\section{Material y métodos}

Área de estudio.- El estudio se realizó en el área protegida del Santuario Nacional los Manglares de Tumbes (SNLMT) (Fig. 1). Ésta área se caracteriza por presentar un bosque de manglar de tipo ribereńo, que se encuentra a lo largo del canal internacional (Perú - Ecuador), influenciado por los estuarios y esteros del río Zarumilla, que viene del Perú y del río Jubones, del Ecuador. El área protegida está formada por diversos sistemas de importancia ecológica, como los manglares, bancos de arena, canales permanentes, etc.; todos ellos influenciados por las mareas (Flores et al. 2013).

En algunas zonas intermareales de los manglares se identificaron parches con la presencia de dos recursos de importancia comercial, Anadara tuberculosa y Ucides occidentalis. En general, en la Zona Baja de Intermareal (ZBI) con presencia del bivalvo $A$. tuberculosa, se observa una baja pendiente, con sedimento fango limoso y con notoria influencia de las mareas. En esta zona se observan las raíces de Rhizophora spp., y también se encuentran otros bivalvos como: A similis, Leukoma aspergina, Mitella guyanensis, Corycorbula sp. Mientras que, en Zona Alta en el Intermareal (ZAI) con presencia del cangrejo Ucides occidentalis, la pendiente es alta, la gente del lugar la conoce como "Altiales", están cubiertas por al menos una vez al mes por las marea más alta o pleamar (Fig. 2).

Muestreos asociados a la ZBI.- En el sitio La Envidia se establecio la Estación 1 ( $3^{\circ} 24^{\prime} 33.70^{\prime \prime S}$; 80² 18'36.30"W) con una extensión de $30 \mathrm{~m}$. Se caracterizó por presentar un sedimento de color negro verdoso. Rhizophora alcanzaba $5 \mathrm{~m}$ de altura aproximadamente. En el área se observó la presencia de cirrípedos pequeńos, ascidias y anemonas hasta la parte media del área, para luego observar $U c a$ spp. y sus madrigueras (Fig. 3A). En Corral de Coches, se estableció la Estación 2 $\left(3^{\circ} 24^{\prime} 36.60 " S ; 80^{\circ} 18^{\prime} 38.10^{\prime \prime W}\right)$ con una extensión de $45 \mathrm{~m}$ de largo se caracterizó por presentar sedimento de color negro verdoso, con árboles de Rhizophora con más de $5 \mathrm{~m}$ de alto,

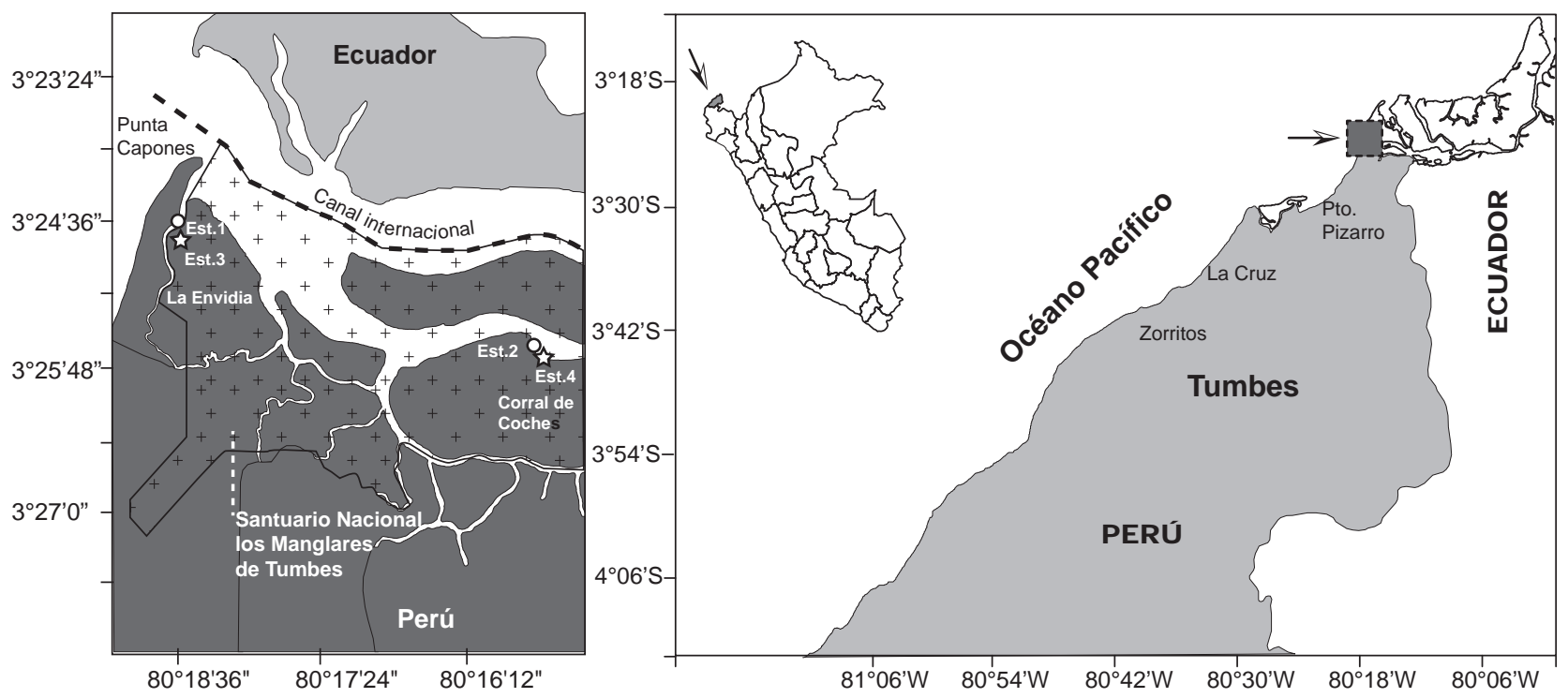

Figura 1. Zona de estudio. En cruces el área protegida del Santuario Nacional los Manglares de Tumbes. En estrellas, las estaciones correspondientes a la Zona Alta del Intermareal (ZAI) y en círculo, las estaciones correspondientes a la Zona Baja del Intermareal (ZBI). 

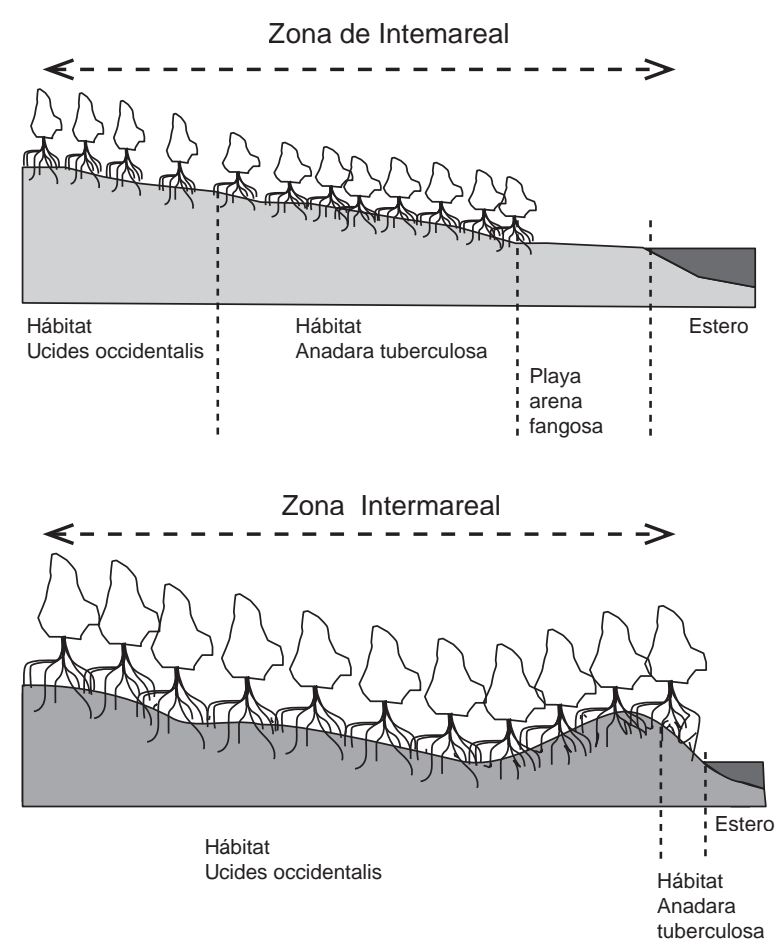

Figura 2. Esquema de la zona de intermareal del manglar del Santuario Nacional los Manglares de Tumbes. Superior) Esquema de la zona baja de intermareal donde hay mayor presencia de Anadara tuberculosa. Inferior) Esquema de la zona alta del intermareal donde hay mayor presencia de Ucides occidentalis en el que domina el hábitat del Cangrejo de Manglar. además se observó la presencia de cirrípedos desde el inicio al final del área estudiada (Fig. 3B).

Muestreos asociados a la ZAI.- En La Envidia se estableció la Estación 3 (324'36.60"S; 80¹8'38.10"W) que se caracterizó por tener un sedimento marrón oscuro y compacto en los primeros metros para luego continuar fangoso (Fig. 3C). En Corral de Coches la Estación 4 (3²5'22.50"S 80¹5'45.00"W) se caracterizó por tener un sedimento marrón rojizo compacto en los primeros metros y luego era fangoso. En ambos sitios, los arboles de Rhizophora fueron viejos, con grandes ramas y troncos en el sedimento. Al colectar la muestra del sedimento en el ZAI hubo una mayor cantidad de raíces en comparación con las colectas en ZBI (Fig. 3D).

Diseño de muestreo.- Los muestreos se realizaron en diciembre de 2012 y marzo de 2013, que correspondian a la temporadas lluviosa.

Dentro del biotopo de manglar propiamente dicho se hicieron transectos perpendiculares al canal del estuario. Se determinaron tres niveles en el transecto. El nivel inferior fue determinado desde el borde del estero en baja hasta la aparición de $A$. tuberculosa, y por la aparicion de las madrigueras de $U$. occidentalis hasta la parte final o nivel superior del transecto, donde no se detectaba la presencia de esta especie. Un nivel medio se ubicó en el centro de cada transecto (Fig. 2 y 4 ).

El muestreo del macrobentos se llevó acabo con un corer $(16 \times 12 \times 30 \mathrm{~cm})$ introducido hasta una aproximado de $30 \mathrm{~cm}$. Se tomaron un total de 72 muestras, 9 réplicas por estación en diciembre de 2012 y marzo de 2013.
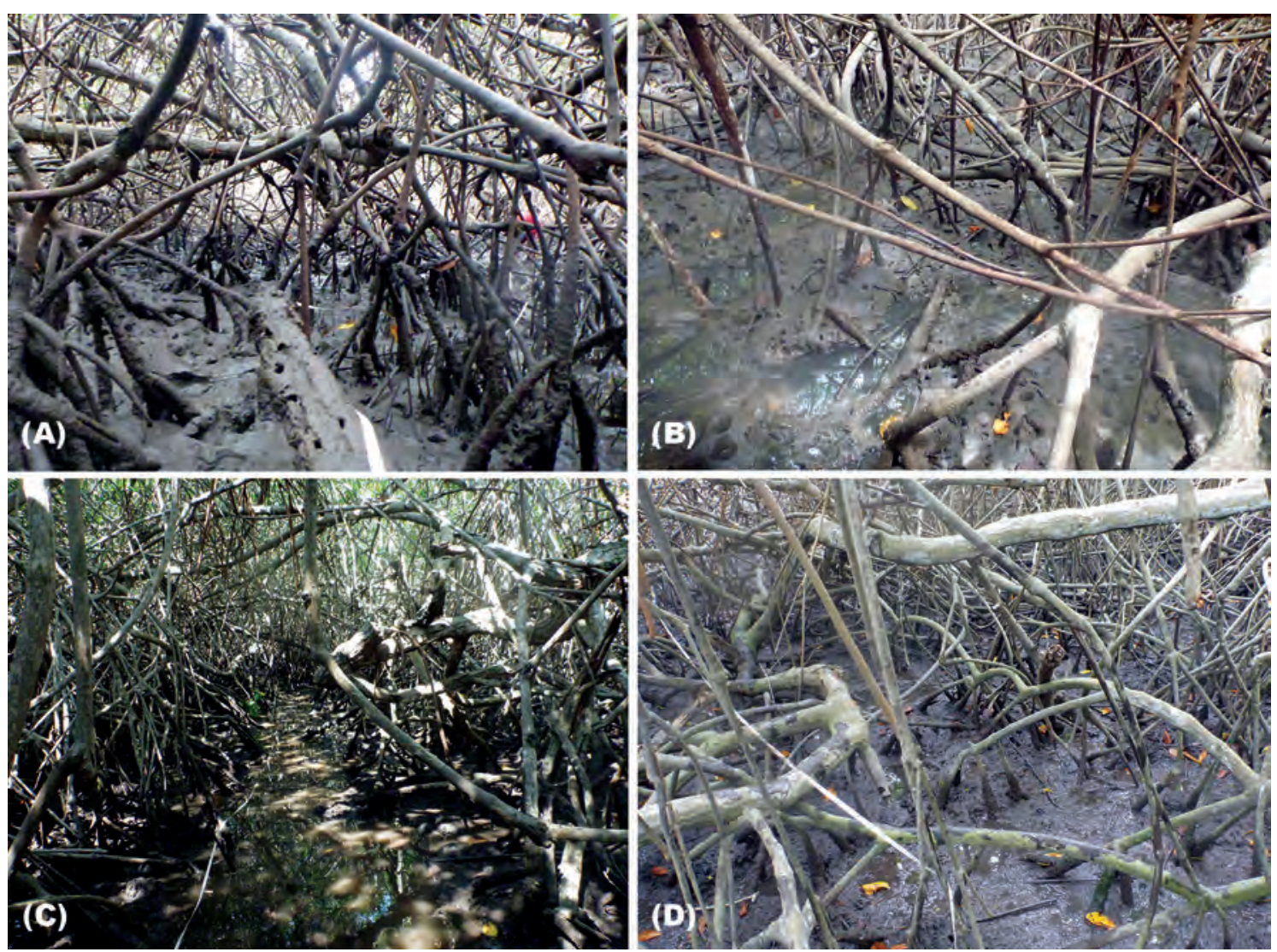

Figura 3. Sitios de nuestros en áreas ZAI y ZBI en el Santuario Nacional los Manglares de Tumbes. Estaciones evaluadas en ZBI (A) Est.1 La Envidia y (B) Est.2 Corral de Coches. Estaciones evaluadas con ZAI (C) Est.3 La Envidia y (D) Est.4 Corral de Coches. 


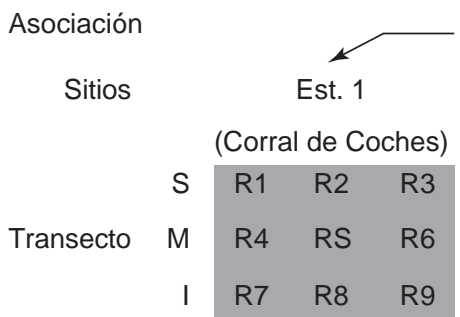

ZBI

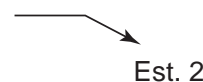

Est. 2

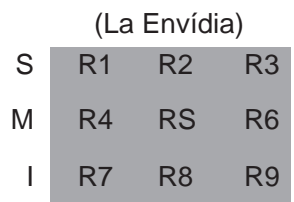

\begin{tabular}{c|ccc}
\multicolumn{4}{c}{ (Corral de Coches) } \\
S & R1 & R2 & R3 \\
M & R4 & RS & R6 \\
I & R7 & R8 & R9
\end{tabular}

ZAI
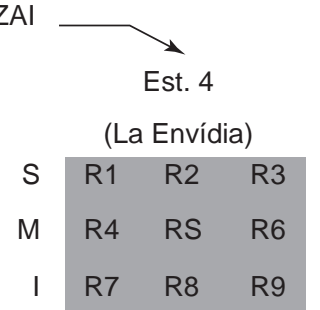

Figura 4. Diagrama de muestreo de macrobentos realizado en el Santuario Nacional los Manglares de Tumbes en la ZAI y ZBI durante diciembre 2012 y marzo 2013. Transecto (S: Superior del transecto, M: Medio del transecto, I: Inferior del Transecto) y Replica (R).

Las muestras de macrobentos obtenidas se tamizaron en el campo con una bolsa tamiz, de abertura de malla de $500 \mu \mathrm{m}, \mathrm{y}$ luego fijadas con formol al 7\%. La separación de los organismos se realizó en laboratorio, con un estereoscopio. La identificación fue al menor taxón posible empleando claves taxonómicas (Fauchald 1977), artículos de táxonomia de poliquetos de Carolina del Norte (Day 1973), familias de poliquetos (Amaral 1980, Elias et al. 2000, Hartamann-Schröder 1959, Rouse \& Pleijel 2007), descripciones de los poliquetos errantes y sedentarios (Day 1967) entre otras publicaciones especializadas; además se realizaron consultas a especialistas.

Los especímenes hallados se agruparon en los seis gremios tróficos: depositívoros superficiales, depositívoros subsuperficiales, carnívoros, herbívoros, filtradores y omnívoros; según la revisión actualizada de Jumars (2015), sobre el artículo de Fauchald \& Jumars, (1979).

El muestreo para la obtención de parámetros fisicoquímicos se realizó en el centro del canal frente a cada transecto con un equipo multiparámetro HORIBA registrándose temperatura, salinidad, $\mathrm{pH}$ y Potencial Redox. Adicionalmente, se obtuvo una muestra de sedimento del nivel medio para la medición con un equipo $\mathrm{PH}$ múltiple portátil WTW registrándose $\mathrm{pH}$ y el potencial Redox.

Análisis de datos.- Se consideraron los principales índices comunitarios: abundancia, biomasa, número de especies, índice de diversidad de Shannon (H') y equidad (J'), según lo descrito por Moreno (2001).

Con la finalidad de comparar la estructura de los ensambles de poliquetos y sus grupos tróficos asociados a las ZAI y ZBI, según la lejanía y cercanía al mar se realizó un análisis de agrupamiento NMDS (Análisis no Paramétrico multidimensional) previa transformación raíz cuarta y la construcción de una matriz de similaridad usando el índice de Bray-Curtis (Clarke \& Gorley, 2006), así también se realizó la construcción de un dendograma de similitud por el método de promedios no ponderados (UPGMA) considerando la presencia y ausencia de especies a partir del índice de Jaccard. Con el fin de determinar diferencias entre los factores de asociación (ZAI y ZBI) y sitio (Corral de coches o lejanía al mar y La Envidia o cercanía al mar) se realizó la prueba de hipótesis ANOSIM (Clarke \& Warwick, 2001).

\section{Resultados}

Parámetros fisicoquímicos.- Los parámetros fisicoquímicos del agua de los canales mostraron ser muy variables, la temperatura del agua varió entre $27-29^{\circ} \mathrm{C}$, la salinidad osciló entre 29 - 32 ppt, el pH fue básico y los valores de Potencial Redox de agua fueron oxidados. Se pudo observar una variación entre los sitios cercanos y lejanos al mar relacionados a la salinidad. En el sedimento los valores de Redox indicaron sedimentos muy reductores en los sitios de la ZAI así también en las estaciones lejanas al mar (Corral de Coches), mientras que el pH del sedimento fue más acida en las estaciones de la ZAI que de la ZBI (Tabla 1).

Composición y Estructura comunitaria.- Se encontró un total de 1373 individuos y 57 especies en todo el estudio. El número de especies fue mayor en las estaciones de la ZBI con 34 especies en la Est. 1 (La Envidia) y 39 en Est. 2 (Corral de los coches). Mientras que en la ZAI, los valores fueron menores, con 22 especies en la Est. 3 (La Envidia) y 20 especies en la Est. 4 (Corral de Coches). Se encontró 30 especies exclusivas la ZBI y 11 para ZAI (Tabla 4).

En general, los valores de densidad y biomasa fueron mayores en la ZBI. De acuerdo a los sitios, la densidad fue mayor en La Envidia en la ZBI (Est. 1), mientras que en Corral de Coches la ZAI (Est. 4) presentó los mayores valores. La biomasa fue mayor en la ZBI de Corral de Coches con mayores (Est. 2), mientras que en la ZAI (Est. 4) de Corral de Coches tuvo la mayor la biomasa (Tabla 2).

El índice $\mathrm{H}^{\prime}$ fue mayor en la ZBI con mayores valores en la Est. 2, mientras que la ZAI, el mayor valor fue en la Est. 4 (Ver Tabla 2). Los mayores valores del índice J' se registraron en la ZAI (Est. 3) de La Envidia y en la ZBI (Est. 2) del Corral de Coches (Tabla 2).

Las familias dominantes en porcentaje de abundancia con $64 \%$ y $16 \%$ respectivamente fueron las familias Capitellidae y Nereididae en la ZAI, mientras que estas mismas familias

Tabla 1. Promedios de los Parámetros fisicoquímicos del agua y el sedimento, registrados en los sitios Corral de coches (lejano al mar) y La Envidia (cercano al mar), en el Santuario Nacional los Manglares de Tumbes.

\begin{tabular}{llcccccc}
\hline \multirow{2}{*}{ Variables } & \multicolumn{2}{c}{ ZBI } & & & \multicolumn{2}{c}{ ZAI } & \\
\cline { 3 - 4 } \cline { 7 - 8 } & & Temperatura $\left({ }^{\circ} \mathrm{C}\right)$ & 27.51 & 29.22 & & 27.52 & 29.26 \\
\multirow{3}{*}{ Agua } & $\mathrm{pH}$ & 7.88 & 7.91 & & 8.61 & 8.17 \\
& Redox $(\mathrm{mV})$ & 184 & 219 & & 222 & 281 \\
& Salinidad $(\mathrm{ppt})$ & 29.88 & 29.01 & & 32 & 29.6 \\
\hline \multirow{2}{*}{ Sedimenton } & Redox & -272.1 & -290.2 & & -280.07 & -298.35 \\
& $\mathrm{PH}$ & 7.15 & 7.30 & & 6.93 & 6.85 \\
\hline
\end{tabular}


Tabla 2. Densidad, Biomasa, Numero de especies (S), Índice de Pielou (J') e Índice Diversidad de Shannon (H') en los meses diciembre 2012 y marzo 2013 en las estaciones de la ZBI y ZBI del Santuario Nacional los Manglares de Tumbes.

\begin{tabular}{|c|c|c|c|c|c|c|c|c|c|c|c|c|}
\hline \multirow[b]{2}{*}{ Asociación } & \multirow[b]{2}{*}{ Est. } & \multirow[b]{2}{*}{ Replica } & \multicolumn{2}{|c|}{ Densidad (ind/m²) } & \multicolumn{2}{|c|}{ Biomasa $\left(\mathrm{g} / \mathrm{m}^{2}\right)$} & \multicolumn{2}{|c|}{$S$} & \multicolumn{2}{|c|}{$\mathrm{J}^{\prime}$} & \multicolumn{2}{|c|}{$\mathbf{H}^{\prime}$} \\
\hline & & & Dic-12 & Mar-13 & Dic-12 & Mar-13 & Dic-12 & Mar-13 & Dic-12 & Mar-13 & Dic-12 & Mar-13 \\
\hline \multirow{18}{*}{ ZBI } & \multirow{9}{*}{1} & R1 & 2656 & 417 & 21.25 & 2.3021 & 10 & 4 & 0.51 & 0.77 & 1.17 & 1.07 \\
\hline & & R2 & 1406 & 1146 & 8.1146 & 7.9427 & 7 & 13 & 0.53 & 0.91 & 1.03 & 2.34 \\
\hline & & R3 & 3385 & 260 & 30.3802 & 0.3646 & 10 & 1 & 0.52 & - & 1.19 & 0 \\
\hline & & R4 & 2656 & 2552 & 19.5313 & 23.8073 & 12 & 12 & 0.77 & 0.82 & 1.9 & 2.03 \\
\hline & & R5 & 885 & 2240 & 32.9167 & 23.2135 & 9 & 11 & 0.88 & 0.79 & 1.93 & 1.9 \\
\hline & & R6 & 729 & 1510 & 17.7969 & 10.8333 & 4 & 8 & 0.72 & 0.76 & 0.99 & 1.58 \\
\hline & & R7 & 1771 & 1354 & 7.1771 & 7.5313 & 14 & 11 & 0.85 & 0.83 & 2.24 & 1.99 \\
\hline & & R8 & 1198 & 1667 & 3.1615 & 5.4635 & 6 & 16 & 0.8 & 0.91 & 1.43 & 2.52 \\
\hline & & R9 & 1510 & - & 7.7396 & - & 13 & - & 0.85 & - & 2.19 & - \\
\hline & \multirow{9}{*}{2} & R1 & 2031 & 573 & 12.901 & 10.9583 & 11 & 8 & 0.75 & 0.95 & 1.81 & 1.97 \\
\hline & & R2 & 625 & 1250 & 0.8594 & 44.3281 & 5 & 11 & 0.84 & 0.83 & 1.35 & 1.98 \\
\hline & & R3 & 1458 & - & 77.3385 & - & 12 & - & 0.87 & - & 2.15 & - \\
\hline & & R4 & 1458 & 2240 & 12.151 & 25.8229 & 8 & 12 & 0.86 & 0.8 & 1.79 & 1.98 \\
\hline & & R5 & 1354 & 2760 & 16.0052 & 27.0417 & 10 & 12 & 0.9 & 0.78 & 2.08 & 1.95 \\
\hline & & R6 & 729 & 1042 & 11.4323 & 1.9844 & 7 & 7 & 0.89 & 0.93 & 1.73 & 1.81 \\
\hline & & R7 & 2969 & 1302 & 11.0104 & 5.6771 & 16 & 10 & 0.91 & 0.84 & 2.53 & 1.92 \\
\hline & & R8 & 2552 & 1771 & 12.0365 & 64 & 12 & 9 & 0.87 & 0.83 & 2.16 & 1.83 \\
\hline & & R9 & 3073 & 1302 & 52.2656 & 19.7969 & 12 & 12 & 0.8 & 0.88 & 2 & 2.19 \\
\hline \multirow{18}{*}{ ZAI } & \multirow{9}{*}{3} & R1 & 260 & 156 & 0.8958 & 6.5677 & 5 & 2 & 1 & 0.92 & 1.61 & 0.64 \\
\hline & & R2 & 156 & - & 4.5729 & - & 3 & - & 1 & - & 1.1 & - \\
\hline & & R3 & - & 625 & - & 0.2344 & - & 3 & - & 0.75 & - & 0.82 \\
\hline & & R4 & 156 & 1042 & 0.6979 & 14.474 & 2 & 6 & 0.92 & 0.79 & 0.64 & 1.41 \\
\hline & & R5 & 104 & 1094 & 0.4427 & 14 & 1 & 6 & - & 0.73 & 0 & 1.31 \\
\hline & & R6 & 104 & 260 & 0.5625 & 1.974 & 2 & 2 & 1 & 0.72 & 0.69 & 0.5 \\
\hline & & R7 & 573 & 104 & 5.7344 & 0.7969 & 6 & 1 & 0.93 & - & 1.67 & 0 \\
\hline & & R8 & - & 208 & - & 0.1719 & - & 2 & - & 0.81 & - & 0.56 \\
\hline & & R9 & 156 & 885 & 2.151 & 1.2969 & 2 & 5 & 0.92 & 0.72 & 0.64 & 1.15 \\
\hline & \multirow{9}{*}{4} & R1 & 2448 & - & 13.1406 & - & 10 & - & 0.78 & - & 1.79 & - \\
\hline & & R2 & 469 & 833 & 1.8906 & 0.8385 & 5 & 4 & 0.95 & 0.59 & 1.52 & 0.82 \\
\hline & & R3 & 52 & - & 0.4219 & - & 1 & - & - & - & 0 & - \\
\hline & & $\mathrm{R} 4$ & - & 156 & - & 0.2083 & - & 1 & - & - & - & 0 \\
\hline & & R5 & 521 & 52 & 10.1146 & 1.4844 & 6 & 1 & 0.95 & - & 1.7 & 0 \\
\hline & & R6 & 208 & 156 & 0.3125 & 0.9583 & 3 & 3 & 0.95 & 1 & 1.04 & 1.1 \\
\hline & & R7 & - & - & - & - & - & - & - & - & - & - \\
\hline & & R8 & 1667 & 625 & 6.9635 & 6.2552 & 8 & 5 & 0.83 & 0.84 & 1.72 & 1.36 \\
\hline & & R9 & 729 & 52 & 7.8906 & 1.5417 & 5 & 1 & 0.78 & - & 1.25 & 0 \\
\hline
\end{tabular}

Tabla 3. Porcentaje de Dominancia de las principales especies según la asociación y los sitios de muestreo del Santuario Nacional los Manglares de Tumbes..

\begin{tabular}{|c|c|c|c|c|c|}
\hline \multicolumn{6}{|c|}{ Zona Baja de Intermareal (ZBI) } \\
\hline & Est.1 & & Est. 2 & & TOTAL \\
\hline Especies & \% Dom & Especies & \%Dom & Especies & $\%$ Dom \\
\hline Notomastus hemipodus & 41.55 & Mediomastus sp. & 26.33 & Notomastus hemipodus & 26.77 \\
\hline Mediomastus sp. & 17.31 & Notomastus hemipodus & 14.29 & Mediomastus sp. & 22.2 \\
\hline Perinereis nuntia & 4.68 & Perinereis nuntia & 8.61 & Perinereis nuntia & 6.81 \\
\hline Linopherus annulata & 4.48 & Schistomeringos annulata & 7.23 & Owenia collaris & 4.94 \\
\hline Heteromastus filiformis & 3.87 & Owenia collaris & 6.02 & Owenia collaris & 4.94 \\
\hline Owenia collaris & 3.67 & Heteromastus filiformis & 5.85 & Schistomeringos annulata & 4.66 \\
\hline Magelona californica & 2.24 & Axiothella rubrocincta & 5.68 & Axiothella rubrocincta & 3.92 \\
\hline \multicolumn{6}{|c|}{ Zona Alta en el Intermareal (ZAI) } \\
\hline & Est. 3 & & Est.4 & & TOTAL \\
\hline Especies & \%Dom & Especies & $\%$ Dom & Especies & $\%$ Dom \\
\hline Mediomastus sp. & 37.17 & Mediomastus sp. & 28.1 & Mediomastus sp. & 31.95 \\
\hline Notomastus hemipodus & 17.7 & Heteromastus filiformis & 16.34 & Heteromastus filiformis & 15.04 \\
\hline Heteromastus filiformis & 13.27 & Capitella capitata & 9.8 & Notomastus hemipodus & 10.9 \\
\hline Perinereis nuntia & 10.62 & Perinereis nuntia & 9.8 & Perinereis nuntia & 10.15 \\
\hline Scoletoma sp. & 3.54 & Prionospio multibranchiata & 9.15 & Capitella capitata & 6.02 \\
\hline Neanthes sp.1 & 2.65 & Notomastus hemipodus & 5.88 & Prionospio multibranchiata & 6.02 \\
\hline Lepidonotus crosslandi & 1.77 & Neanthes sp.1 & 4.58 & Neanthes sp.1 & 3.76 \\
\hline
\end{tabular}


Tabla 4. Relación de las Especies por las Asociaciones y zonas de muestreo del Santuario Nacional los Manglares de Tumbes. En paréntesis el gremio trófico: Ds: Depositívoros Superficiales, Dss: Depositívoros SubSuperficiales, Ca: Carnívoros, Fi: Filtradores, He: Herbívoros, Om: Omnívoros.

\begin{tabular}{|c|c|c|c|c|}
\hline \multirow{2}{*}{ Especie, Grupo trófico } & \multicolumn{2}{|c|}{ Corral Coches } & \multicolumn{2}{|c|}{ La Envidia } \\
\hline & ZBI & ZAI & ZBI & ZAI \\
\hline Axiothella rubrocincta (Ds) & $\mathrm{X}$ & $\mathrm{X}$ & $X$ & \\
\hline Capitella capitata (Ds) & $x$ & $x$ & $x$ & $x$ \\
\hline Capitella sp. (Ds) & $x$ & & $x$ & \\
\hline Cossura laeviseta (Ds) & & & $x$ & \\
\hline Heteromastus filiformis (Ds) & $X$ & $X$ & $x$ & $x$ \\
\hline Leitoscoloplos foliosus (Ds) & $X$ & & $x$ & \\
\hline Leitoscoloplos kergelensis (Ds) & $x$ & & $x$ & \\
\hline Mediomastus sp. (Ds) & $X$ & $X$ & $x$ & $x$ \\
\hline Neomediomastus sp. (Ds) & & & $x$ & \\
\hline Notomastus hemipodus (Ds) & $x$ & $x$ & $x$ & $x$ \\
\hline Notomastus lineatus (Ds) & & & $x$ & \\
\hline Pectinaria chilensis (Ds) & $X$ & & $x$ & $x$ \\
\hline Chaetozone sp. (Dss) & $x$ & & $x$ & \\
\hline Chaetozone sp.1 (Dss) & & $x$ & & $X$ \\
\hline Isolda pulchella (Dss) & $x$ & $x$ & $x$ & $X$ \\
\hline Lanice sp. (Dss) & & & $x$ & $X$ \\
\hline Magelona californica (Dss) & & & $x$ & \\
\hline Prionospio multibranchiata (Dss) & $x$ & $x$ & $x$ & $X$ \\
\hline Prionospio sp. (Dss) & & & & $x$ \\
\hline Trochochaeta cf. mexicana (Dss) & $X$ & & $x$ & \\
\hline Dipolydora socialis (Ds, Dss) & $x$ & $x$ & $x$ & $x$ \\
\hline Polydora sp. (Ds, Dss) & & & & $x$ \\
\hline Spiophanes duplex (Ds, Dss) & & & $x$ & \\
\hline Ancistrosyllis hamata (Ca) & & & $x$ & \\
\hline Arabella sp. (Ca) & $x$ & & & \\
\hline Chrysopetalidae (Ca) & & & $x$ & \\
\hline Exogone lourei (Ca) & & & $x$ & \\
\hline Harmothoe sp. (Ca) & $x$ & & & \\
\hline Hermundura fauveli $(\mathrm{Ca})$ & $x$ & & $x$ & $x$ \\
\hline Lepidonotidae $2(\mathrm{Ca})$ & & $X$ & & \\
\hline Lepidonotus crosslandi (Ca) & $x$ & $X$ & $x$ & $x$ \\
\hline Linopherus annulata (Ca) & & & $x$ & \\
\hline Linopherus fauchaldi (Ca) & & & $x$ & \\
\hline Linopherus paucibranchiata (Ca) & $x$ & & & \\
\hline Linopherus sp. (Ca) & & & $x$ & \\
\hline Marphysa sp. (Ca) & $x$ & & & \\
\hline Nephtys cf. gabra (Ca) & $x$ & & & \\
\hline Oxydromus sp. (Ca) & $x$ & & $x$ & $x$ \\
\hline Phyllodoce sp. (Ca) & $X$ & & $x$ & \\
\hline Podarkeopsis brevicephala $(\mathrm{Ca})$ & & $X$ & & \\
\hline Podarkeopsis brevipalpa (Ca) & $x$ & & $x$ & \\
\hline Podarkeopsis sp. (Ca) & & $x$ & & \\
\hline Schistomeringos annulata (Ca) & $x$ & $x$ & $x$ & \\
\hline Scoletoma sp. (Ca) & $x$ & $x$ & $x$ & $x$ \\
\hline Sigambra tentaculata $(\mathrm{Ca})$ & $x$ & $x$ & & \\
\hline Synelmis albini $(\mathrm{Ca})$ & $x$ & & $x$ & \\
\hline Aracia sp. (Fi) & $x$ & & & \\
\hline Oligochaeta $(\mathrm{Fi})$ & $x$ & & $x$ & \\
\hline Owenia collaris $(\mathrm{Fi})$ & $x$ & & $x$ & \\
\hline Parasabella leucaspis (Fi) & $x$ & & & \\
\hline Sabelaria gracilis $(\mathrm{Fi})$ & $x$ & & & \\
\hline Lysidice sp. (He) & & & $x$ & \\
\hline Neanthes sp.1 (Om) & & $x$ & $x$ & $x$ \\
\hline Neanthes sp.9 (Om) & & $x$ & & $x$ \\
\hline Nereis cf. oligoalina $(\mathrm{Om})$ & & & & $X$ \\
\hline Perinereis cf. rookeri $(\mathrm{Om})$ & & $x$ & & \\
\hline Perinereis nuntia (Om) & $x$ & $x$ & $x$ & $X$ \\
\hline Rullierinereis sp. (Om) & & $x$ & & \\
\hline Syllis sp. $(\mathrm{Om})$ & & $x$ & & \\
\hline
\end{tabular}

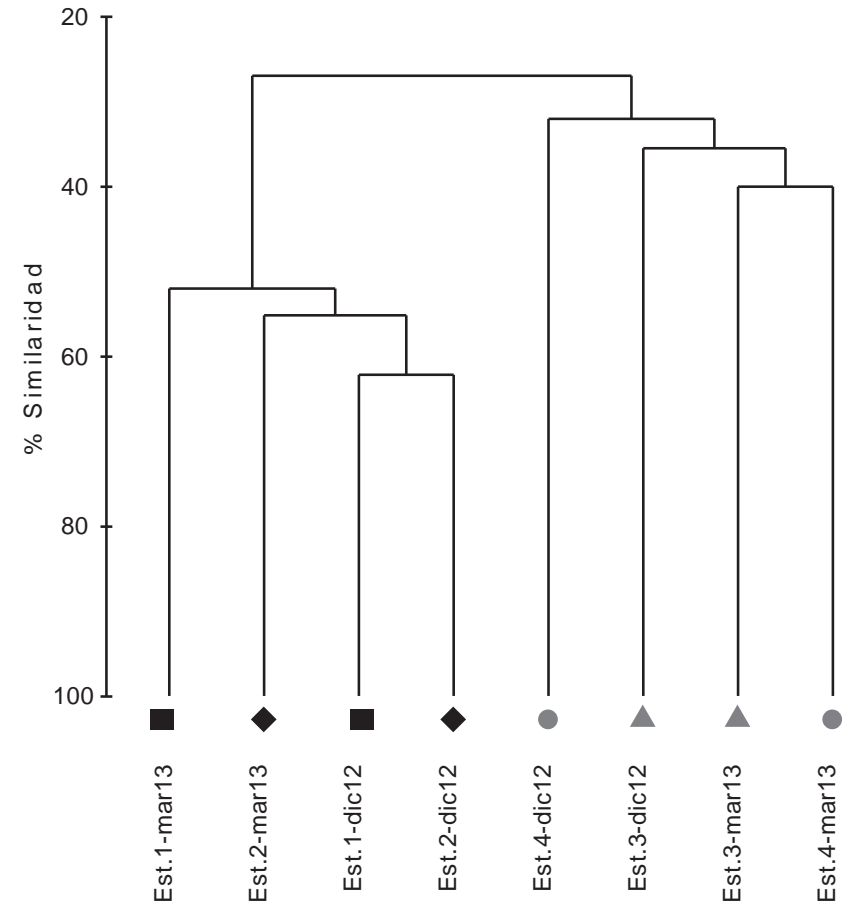

Figura 5. Análisis de Dendograma basada en presencia y ausencia de especies de poliquetos en la ZBI y ZAI del Santuario Nacional los Manglares de Tumbes. La ZBI en negro con la estaciones 1 en cuadrado y 2 y rombo respectivamente. EI ZAI en gris con las estaciones 3 y 4 en triángulo y círculo respectivamente.

obtuvieron abundancias del $55 \%$ y $6 \%$ respectivamente en la ZBI. La familia con mayor número de especies se encontró en ZBI es la familia Capitellidae (7 especies) mientras que en ZAI fue la familia Nereididae (5 especies) (Tabla 3).

Entre las especies dominantes en abundancia en la ZAI se encuentran los capitélidos Mediomastus ambiseta, Heteromastus filiformis y Notomastus hemipodus (alternando su dominancia entre los sitios), mientras que, en la ZBI las especies dominantes son los capitélidos $N$. hemipodus, $M$. ambiseta y el nereido Perinereis nuntia (presentes en ese orden por sitios) (Tabla 3). La frecuencia de ocurrencia de las especies con más del $50 \%$ se encontró en la ZAI con Mediomastus ambiseta y Heteromastus filiformis mientras que en la ZBI encontramos a $N$. hemipodus, M. ambiseta, y H. filiformis, P. nuntia, O. collaris y Schistomeringos annulata.

El Análisis de similitud por presencia y ausencia de especies permitió identificar dos grupos de ensambles de poliquetos pertenecientes a ZAI e ZBI (Fig. 5). El grupo de poliquetos en ZBI presenta una similitud del más del $50 \%$ no encontrándose diferencias entre sitios, mientras que en ZAI se encontró una baja similitud entre los sitios.

El análisis de escalamiento multidimensional no paramétrico (NMDS) a partir de las abundancias promedios muestra una clara separación entre los ensambles de poliquetos en las la ZBI y de la ZAI (Fig. 6). La prueba ANOSIM de una Via encontró diferencias significativas entre los sitios con cierto solapamiento $(\mathrm{p}<0.05 ; \mathrm{R}=0.5)$. Al comparar los ensambles de poliquetos en ZAI e ZBI se encontró diferencias significativas $(\mathrm{p}<0.05 ; \mathrm{R}=0.813)$. 


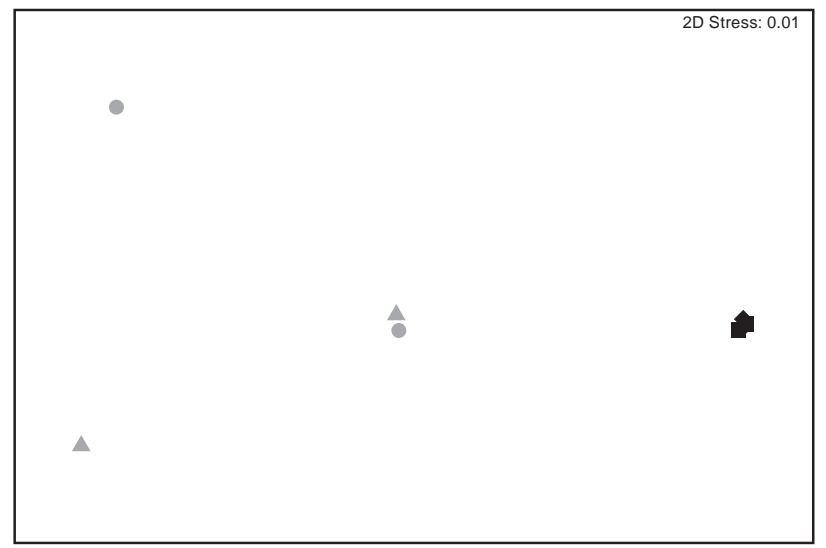

Figura 6. Escalamiento multidimensional no paramétrico (NMDS) con transformación raíz cuarta basada en las abundancias de las especies de poliquetos de ZBI y ZAI del Santuario Nacional los Manglares de Tumbes. La ZBI en negro con la estaciones 1 y 2 en cuadrado y rombo respectivamente. El ZAI en gris con las estaciones 3 y 4 en triangulo y círculo respectivamente.

Gremios tróficos.- Los gremios tróficos se encontraron representados por los carnívoros, depositívoros superficiales, depositívoros subsuperficiales, filtradores, herbívoros y omnívoros. En la ZBI se hallaron los seis gremios tróficos en La Envidia y cinco en Corral de Coches, mientras que en la ZAI se hallaron cuatro gremios tróficos en ambos lugares (Fig. 7). Se observó mayor presencia de taxones del grupo carnívoros, los que cuentan con 20 representantes entre la ZBI y ZAI. Por otro lado, el grupo dominante en abundancia fue depositívoros superficiales en ambas zonas (Tabla 5).

Para la ZBI se encontró 14 representantes carnívoros en Corral de Coches y 14 en La Envidia, en la ZAI 7 especies carnívoras estuvieron presentes en Corral de coches y solo 4 en la Envidia, solo en este último caso el grupo carnívoros quedó relegado al segundo lugar, siendo los depositívoros

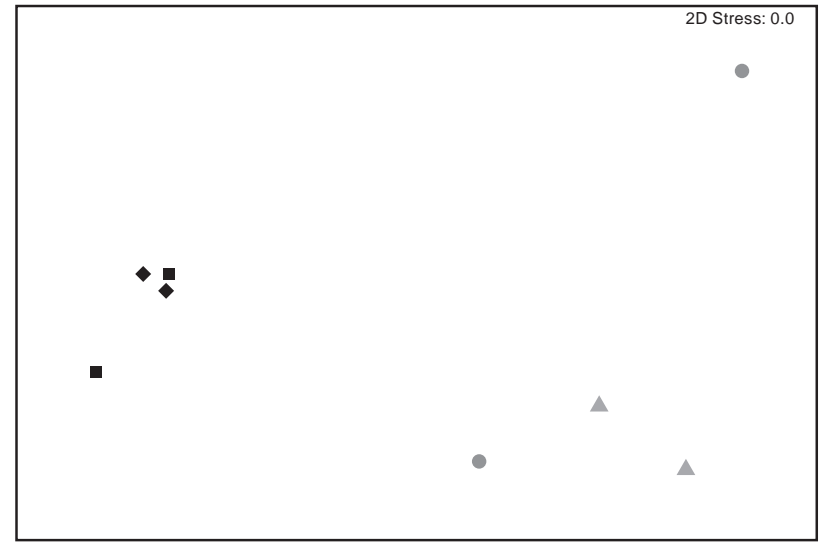

Figura 8. Escalamiento multidimensional no paramétrico (NMDS) con transformación a raíz cuarta de la distribución de especies según los Grupos Tróficos de ZBI y ZAI del Santuario Nacional los Manglares de Tumbes. Estación 1 y 2 (Cuadrado y Rombo) corresponde a ZBI y la estación 3 y 4 (triángulo y círculo) a ZAI.

subsuperficiales los más representativos de dicha zona. A pesar de la dominancia de los carnívoros, la distribución de los grupos tróficos fue diferente entre ambas asociaciones, con una distribución muy similar, con los mismos gremios tróficos en orden de dominancia en la ZBI tanto en Corral de Coches como en La Envidia, mientras que en la ZAI la distribución fue heterogénea (Tabla 5).

En el Figura 8 (NMDS), se pueden observar diferencias entre la distribución de especies por grupo trófico, con un agrupamiento más compacto entre las muestras pertenecientes a la ZBI, mientras que las muestras pertenecientes a la ZAI se muestran más dispersas. Mediante la prueba ANOSIM se observaron diferencias significativas entre zonas $(\mathrm{R}=0.89 ; \mathrm{p}=0.02)$ y entre estaciones $(R=0.52 ; \mathrm{p}=0.08)$.

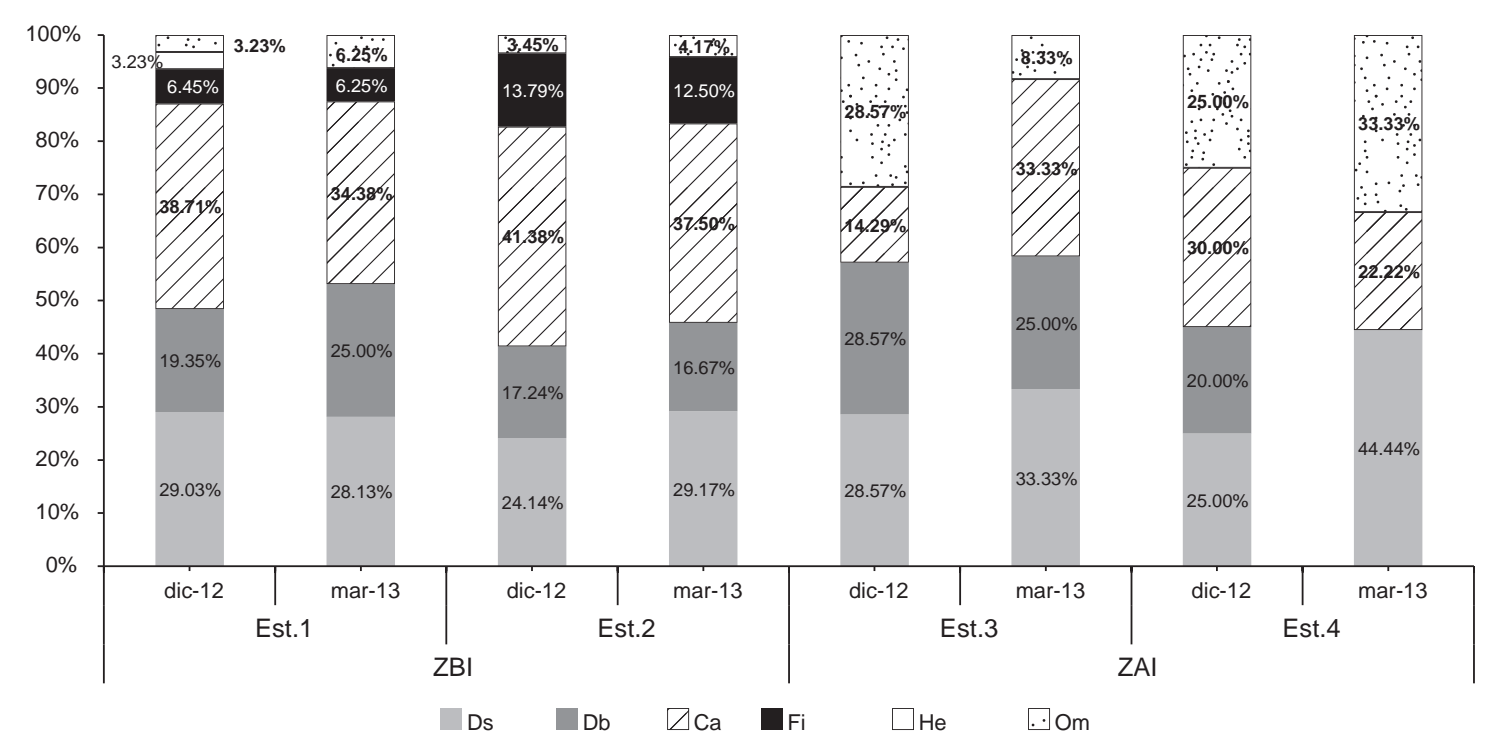

Figura 7. Distribución porcentual de gremios tróficos de los ensambles de poliquetos de ZBI Y ZAI del SNLMT. Ds = Depositívoros superficiales; $\mathrm{Db}=$ Depositívoros subsuperficiales; $\mathrm{Ca}=$ Carnívoros; $\mathrm{Fi}=$ Filtradores; He=Herbívoros; Om = Omnívoros. 
Tabla 5. Número de individuos y especies por gremio trófico en las estaciones muestreadas. Dss: Depositívoros subsuperficiales; Ds: Depositívoros superficiales; Ca: Carnívoros; Fi: Filtradores; He: Herbívoros; Om: Omnívoros

\begin{tabular}{|c|c|c|c|c|c|}
\hline \multirow[t]{2}{*}{ Estaciones } & \multirow[t]{2}{*}{ Gremios tróficos } & \multicolumn{2}{|c|}{ Abundancia } & \multicolumn{2}{|c|}{$\mathbf{N}^{\circ}$ Especies } \\
\hline & & Dic-12 & Mar-13 & Dic-12 & Mar-13 \\
\hline \multirow[t]{6}{*}{ Est.1 } & Depositívoros subsuperficiales & 24.0 & 14.7 & 9 & 9 \\
\hline & Depositívoros superficiales & 2.0 & 2.3 & 6 & 8 \\
\hline & Carnívoros & 5.7 & 4.7 & 12 & 11 \\
\hline & Filtradores & 1.3 & 2.6 & 2 & 2 \\
\hline & Herbívoros & 0.1 & 0.0 & 1 & 0 \\
\hline & Omnívoros & 2.0 & 0.8 & 1 & 2 \\
\hline \multirow[t]{6}{*}{ Est.2 } & Depositívoros subsuperficiales & 18.2 & 16.2 & 7 & 7 \\
\hline & Depositívoros superficiales & 1.9 & 1.9 & 5 & 4 \\
\hline & Carnívoros & 7.3 & 5.4 & 12 & 9 \\
\hline & Filtradores & 3.6 & 2.8 & 4 & 3 \\
\hline & Herbívoros & 0.0 & 0.0 & 0 & 0 \\
\hline & Omnívoros & 4.0 & 1.6 & 1 & 1 \\
\hline \multirow[t]{6}{*}{ Est.3 } & Depositívoros subsuperficiales & 1.2 & 7.6 & 4 & 4 \\
\hline & Depositívoros superficiales & 0.4 & 0.4 & 4 & 3 \\
\hline & Carnívoros & 0.4 & 0.6 & 2 & 4 \\
\hline & Filtradores & 0.0 & 0.0 & 0 & 0 \\
\hline & Herbívoros & 0.0 & 0.0 & 0 & 0 \\
\hline & Omnívoros & 1.1 & 0.8 & 4 & 1 \\
\hline \multirow[t]{6}{*}{ Est.4 } & Depositívoros subsuperficiales & 7.1 & 3.2 & 5 & 4 \\
\hline & Depositívoros superficiales & 2.1 & 0.0 & 4 & 0 \\
\hline & Carnívoros & 1.3 & 0.2 & 6 & 2 \\
\hline & Filtradores & 0.0 & 0.0 & 0 & 0 \\
\hline & Herbívoros & 0.0 & 0.0 & 0 & 0 \\
\hline & Omnívoros & 2.4 & 0.6 & 5 & 3 \\
\hline
\end{tabular}

\section{Discusión}

La composición del ensamble de poliquetos se encuentra con mayores valores de abundancia, biomasa, número de especies e índices de diversidad en la ZBI, al parecer estaría relacionado a la localización del biotopo, es decir al nivel bajo del intermareal, que se encuentra influenciada por la frecuencia de inundación de la marea, esto facilita el reclutamiento de poliquetos (Metcalfe \& Glasby 2008), incluso características del biotopo como la presencia de las conchas vacías de los moluscos muertos favorecerían por el refugio que proporcionarían a los poliquetos (Gutierrez et al. 2003).

En el caso de la composición de ZAI, se encontró los valores más bajos en abundancia, biomasa, número de especies e índice H' en comparación a ZBI. La poca abundancia de la poliquetofauna en la ZAI estaría asociada a la desecación (Metcalfe \& Glasby 2008, Ulrich Saint \& Schneider 2010), ya que se encuentra en las zonas altas comúnmente llamada "altiales" por los extractores de Cangrejo de manglar. Además, en esta zona, encontramos raíces viejas de Rhizophora mangle con alta producción de taninos que podrían inhibir el crecimiento de comunidades bentónicas como se ha reportado en otros manglares (Morrisey et al., 2010).

La relación abundancia/biomasa entre los sitios fue diferente.
En la ZBI de La Envidia, cercana a la desembocadura al mar, los altos valores de abundancia y baja biomasa, podrían relacionarse a procesos de reclutamiento. En cambio, la ZAI de Corral de Coches, estación lejana del mar, presentó valores de abundancia y biomasa altos.

El número de especie fue similar entre los sitios. Esto coincide con estudios en estuarios subtropical en el que el número de especies no presenta variación espacial con respecto a la cercanía o lejanía al mar (Morais et al. 2016).

La familia Capitellidae fue la más diversa en el presente estudio. Esta familia está relacionada a la descomposición de materia orgánica en sedimentos, como ha sido señalado en diferentes estudios en las zonas intermareales de manglares tropicales y templados (Metcalfe \& Glasby 2008, Morales 2012, Nordhaus et al. 2009). Por ejemplo, la dominancia del capitelido Notomastus hemipodus indica sedimentos altamente reductores, estudios sobre este género indican que se caracteriza por su poco desplazamiento, y su adaptación a la hipoxia (Kikuchi, 1987). El nereido Perinereis nuntia, también dominante en el presente estudio, ha sido reportado como tolerante a las variaciones de salinidad, hipoxia y es resistente a contaminantes (Miron \& Kristensen 1993, Won et al. 2012).

Así también, la mayor presencia de gremios tróficos con mayor número de especies en la ZBI, en las dos estaciones nos 
indica un biotopo mas resiliente, sin embargo la presencia de varios taxa carnívoros nos indicaría la importancia del stress biológico por depredación, típico de áreas de baja marea (Dittmann, 1996; Reise, 1985). Como se esperaba el gremio de depositívoros dominó (Sarkar et al. 2005), además, la dominancia de los depositívoros superficiales también podría indicar un hábitat con alto grado de perturbación (Metcalfe \& Glasby 2008), por lo que se podría considerar a la ZAI como un hábitat con mayor perturbación respecto a la ZBI.

Los análisis multivariados demostraron que la estructura del ensamble y sus gremios tróficos de la ZAI e ZBI, son distintas, esto por la presencia de especies exclusivas en cada sitio estudiado. Así mismo, el análisis multivariado también nos permite observar una alta dispersión de los ensambles de las estaciones de ZAI, dando indicios de variabilidad a pequeńa escala, causado al parecer por un mayor stress fisicoquímico e impacto antropogénico (v. g. actividad extractiva) (Lee 2008).

Por lo contrario a ZBI, en el análisis multivariado se muestra menor variabilidad y dispersión, sin cambios en las estaciones con respecto a la estructura del ensamble de poliquetofauna, al parecer, el constante reclutamiento y características del hábitat sumada a más presencia de grupos tróficos proporcionaría un ensamble de poliquetos similares con menos variabilidad espacial y más resiliencia a cambios ambientales.

En conclusión se encontró que la composición y grupos tróficos de las asociaciones de poliquetofauna son diferentes, explicados en parte por las características del biotopo. Así pues, con algunas peculiaridades de las asociaciones por sitios al parecer no se puede descartar estar influenciada por variaciones espaciales.

A pesar de estas diferencias, en ambas asociaciones dominan las mismas familias (Capitellidae y Nereididae) las cuales alternaron sus especies dominantes presentando una diferente distribución. Así pues, el análisis multivariado no pudo diferenciar las dos asociaciones ni sus sitios, sin embargo se pudo observar que la asociación ZAI presenta una mayor variabilidad.

\section{Agradecimiento}

Nosotros agradecemos al proyecto "Impacto de la Variabilidad y Cambio climático en los Manglares de Tumbes", financiado por el IRDC. A los integrantes del laboratorio de Ecología Marina de la UNMSM. Un especial agradecimiento al Dr. Juan Tarazona Barboza por su apoyo en la realización del proyecto.

\section{Literatura citada}

Amaral A. C. Z. 1980. Breve caracterização dos gêneros da familia Capitellidae Grube (Annelida, Polychaeta) e descriçáo de Nonatus longilineus gen. sp. nov. Boletim do Instituto Oceanográfico 29 (1): 99-106. doi:10.1590/S037355241980000100008 .

Day, J.H. 1973. New polychaeta from Beaufort, with a key to all species recorded from North Carolina. NOAA Technical Reports, Ser. National Marine Fisheries Service, Circulars, 375: 1-140. doi: http://doi.org/10.5962/bhl.title.62852

Clarke K.R. \& R.N. Gorley. 2006. PRIMER v6: User Manual/Tutorial. PRIMER-E, Plymouth, 192pp.

Clarke K.R. \& R.M. Warwick. 2001. Change in marine communities: an approach to statistical analysis and interpretation, 2nd edition. PRIMER-E, Plymouth, 172pp.

Day J. H. 1967. A Monograph on the Polychaeta of Southern Africa Part 1, Errantia: Part 2, Sedentaria Published by the Trustees of the British Museum (Natural History), London, 1967 Publication no. 656. Pp. viii +878 .
Dittmann S. 1996. Effects of macrobenthic burrows on infaunal communities in tropical tidal flats. Marine Ecology Progress Series, 134, 119-130. doi: http://doi.org/10.3354/ meps134119

Duke, N. (1993). Mangrove floristics and biogeography. Tropical Mangrove Ecosystems, 41, 63 - 100. Doi: http://doi. org/10.1029/CE041p0063

DS N ${ }^{\circ}$ 018-88-AG. 1988. Declaran Santuario Nacional superficie ubicada en Zarumilla, Tumbes. El Peruano, Normas Legales, 7 de marzo de 1988.

Echevarría J. \& Sarabia, J. 1993. Manglares en el Perú. In Conservación y aprovechamiento sostenible de los bosques de manglar en las regiones América Latina y Africa (Proyecto I, pp. 39-48). Lima-Perú: International Tropical Timber Organization.

Elías R., E. Alberto Vallarino \& C.S. Bremec. 2000. Protoariciella uncinata Hartmann-Schröeder, 1962 (Polychaeta, Orbiniidae): a new record for intertidal mussel beds of the Southwestern Atlantic shore affected by sewage effluents. Revista de biología marina y oceanografía 35 (2): 181-84.

Fauchald K. 1977. The polychaete worms, definitions and keys to the orders, families and genera. Natural History Museum of Los Angeles County: Los Angeles, CA (USA), Science Series. 28:1-188.

Fauchald, K., \& Jumars, P. (1979). The diet of worms: a study of polychaete feeding guilds. Oceanography and Marine Biology. An Annual Review, 17, 193-284.

Flores D., L. Cespedes \& A. Martinez. 2013. Identificación de Servicios Ecosistémicos en el Santuario Nacional Los Manglares de Tumbes, Informe tecnico final. Instituto Geofísico del Perú. Lima. 84 pp.

Gilman E.L., J. Ellison, NC.Duke \& C. Field. 2008. Threats to mangroves from climate change and adaptation options: A review. Aquatic Botany, Mangrove Ecology - Applications in Forestry and Costal Zone Management, 89 (2): 237-50. doi: http://doi.org/10.1016/j.aquabot.2007.12.009.

Giangrande A., M. Licciano \& L. Musco. 2005. Polychaetes as Environmental Indicators Revisited. Marine Pollution Bulletin 50 (11): 1153-62. doi: http://doi.org/10.1016/j. marpolbul.2005.08.003.

Gutierrez J.L., C.G. Jones, D.L. Strayer \& O.O. Iribarne. 2003. Mollusks as ecosystem engineers: the role of shell production in aquatic habitats. Oikos, 101: 79-90. Doi: http://doi. org/10.1034/j.1600-0706.2003.12322.x

Hartmann-Schröder G. 1959. Zur ökologie der polychaeten des mangrove-estero-gebietes von El salvador». Beitrage zur Neotropischen Fauna 1 (2): 69-183. doi: http://doi. org/10.1080/01650525909380612.

Hutchings P.A \& Recher H.F. 1982. The fauna of Australian Mangroves. Proceedings of The Linnean Society of New South Wales 106. 83-121.

INRENA. (2007). Plan Maestro del Santuario Nacional Los Manglares de Tumbes 2007 - 2011. Instituto Nacional de Recursos Naturales - INRENA. Lima. 197 pp.

Jumars P.A, K.M. Dorgan \& S.M. Lindsay. 2015. Diet of worms emended: an update of polychaete feeding guilds. Annual Review of Marine Science, 7(July 2014), 497-520. http:// doi.org/10.1146/annurev-marine-010814-020007

Kikuchi E. 1987. Effects of the brackish deposit-feeding polychaetes Notomastus sp. (Capitellidae) and Neanthes japonica (Izuka) (Nereidae) on sedimentary $\mathrm{O} 2$ consumption and CO2 production rates. Journal of Experimental Marine Biology and Ecology, 114, 15-25. doi: http://doi.org/10.1016/00220981(87)90136-5

Lacerda LD. [coordinador] 1993. Conservación y aprovechamiento sostenible de bosques de manglar en las regiones America Latina y Africa, informe técnico del proyecto. Parte 1: América Latina. ITTO Technical Series (Japón). International Society for Mangrove Ecosystems, Okinawa (Japón). Okinawa. 256 pp.

Lee S.Y. 2008. Mangrove macrobenthos: Assemblages, services, and linkages. Journal of Sea Research, Mangrove Macrobenthos Special IssueProceedings of the Mangrove Macrobenthos Meeting II, 59 (1-2): 16-29. doi:10.1016/j. seares.2007.05.002. 
Macnae W. 1968. A general account of the fauna and flora of mangrove swamps and forest in the Ondo-west Pacific region. Advances in Marine Biology, 6, 73-270. doi: http://doi.org/10.1016/ S0065-2881(08)60438-1

Metcalfe KN. \& CJ. Glasby. 2008. Diversity of Polychaeta (Annelida) and other worm taxa in mangrove habitats of Darwin Harbour, northern Australia. Journal of Sea Research 59, 70-82. doi: http://doi.org/10.1016/j.seares.2007.06.002

Miron G. \& E. Kristensen. 1993. Factors influencing the distribution of nereid polychaetes: the sulfide aspect. Marine Ecology Progress Series, 93(1990), 143-153. Doi: http://doi. org/10.3354/meps093143

Morais G.C., M.G. Camargo \& P. Lana. 2016. Intertidal assemblage variation across a subtropical estuarine gradient: How good conceptual and empirical models are? Estuarine, Coastal and Shelf Science, 170, 91-101. http://doi.org/10.1016/j. ecss.2015.12.020

Morales J. de D. 2012. Diversidad y abundancia de macroinvertebrados en manglares naturales y restaurados del noroeste de Ecuador. Tesis (Licenciado en Ecología Aplicada), Universidad San Francisco de Quito, Colegio de Ciencias Biológicas y Ambientales; Quito. http://repositorio.usfq. edu.ec/handle/23000/1811

Moreno C.E. 2001. Métodos para medir la biodiversidad. M\&T Manuales Y Tesis SEA, 1, 84

Morrisey D., A. Swales, S. Dittmann, M.A. Morrison, C.E. Lovelock \& C.M. Beard. 2010. The Ecology and Management of Temperate Mangroves. Oceanography and Marine Biology: An Annual Review, 48(322), 43-160. http://doi.org/10.1201/ EBK1439821169-c2
Nordhaus I., F.A. Hadipudjana, R. Janssen \& J. Pamungkas. 2009. Spatio-temporal variation of macrobenthic communities in the mangrove-fringed Segara Anakan lagoon, Indonesia, affected by anthropogenic activities. Regional Environmental Change, 9(4), 291-313. http://doi.org/10.1007/ s10113-009-0097-5

Peña, M. (1970). Biocenosis de los Manglares Peruanos. Separata de La Revista Anales Científicos, IX, 38-45.

Reise K. 1985. Tidal Flat Ecology. Springer, Berlin, 191pp.

Rouse G.W., \& F. Pleijel. 2007. Annelida. Zootaxa, 264, 245-264.

Sarkar S.K., A. Bhattacharya, S. Giri, A. Bhattacharya, D. Sarkar, D.C. Nayak \& A.K. Chattopadhaya. 2005. Spatiotemporal variation in benthic polychaetes (Annelida) and relationships with environmental variables in a tropical estuary. Wetlands Ecology and Management, 13: 55-67. http://doi. org/10.1007/s11273-003-5067-y

Takahashi K. \& Martínez, A. 2015. Impacto de la Variabilidad y Cambio Climático en el Ecosistema de Manglares de Tumbes, Perú. IDRC \#106714-001 (CCW). Instituto Geofísico del Perú. Lima.

Ulrich Saint P. \& H. Schneider, (Eds). 2010. Mangrove Dinamics and Management in North Brazil. Ecological Studies vol 211, Berlin, Heidelberg: Springer Berlin Heidelberg. 114 pp.

Vargas Rodriguez N.N.M. 1993. Comunidades intermareales macrozzoobeticas en los manglares de Puerto Pizarro-Tumbes. Tesis para optar el Titulo Profesional de Licenciada en Biología. Facultad de Ciencias Biológicas, Universidad Ricardo Palma. Lima. 79 pp, 15 Figs. 15 Cuadros.

Won E.J., J.S. Rhee, R.O. Kim, K. Ra, K.T. Kim, K.H. Shin \& J.S. Lee. 2012. Susceptibility to oxidative stress and modulated expression of antioxidant genes in the copper-exposed polychaete Perinereis nuntia. Comparative Biochemistry and Physiology - C Toxicology and Pharmacology, 155(2), 344-351. Doi: http://doi.org/10.1016/j.cbpc.2011.10.002 